\title{
Die kommunikativ-pragmatische Orientierung im Fremdsprachenunterricht und ihre theoretische Grundlegung - Kritik und Perspektiven aus glottodidiaktischer Sicht
}

Theoretical Background of Communicative-Pragmatic Approaches

to Foreign Language Teaching - criticism and perspectives

from a glottodidactic point of view

Barbara SADOWNIK ${ }^{1}$

Uniwersytet Marii Curie-Skłodowskiej w Lublinie

\begin{abstract}
The aim of the paper is to discuss theoretical assumptions as well as point out strengths and weaknesses of the communicative-pragmatic approach to foreign language teaching. The author underlines the fact that pragmatics and the theory of speech acts by J. Austin and J. Searle, which emerged under the influence of the pragmatic approach in American philosophy, sociology and semiotics, as well as the theory of language games by L. Wittgenstein, the notion of communicative competence by D. Hymes and the theory of communicative action by $\mathrm{J}$. Habermas all influenced the proponents of the pragmatic-communicative approach either directly or indirectly. The implementation of these theories, however, has not always been cautious and critical enough, which is exemplified by, for instance, overemphasizing fluency and communicative efficiency over morphosyntactic accuracy of speech. In other words, changing

(- https://orcid.org/0000-0003-1528-7975

Uniwersytet Marii Curie-Skłodowskiej w Lublinie/Marie Curie-Sklodowska University in Lublin

barbara.sadownik@poczta.umcs.lublin.pl
\end{abstract}


the role of communicative competence and downgrading the significance of linguistic competence in the foreign language teaching process. The author concludes that the essence of natural languages lies in their immanent structure rather than the communicative purposes they serve.

Keywords: Communicative-pragmatic approach, glottodidactics, generative grammar, speech acts theory, communicative-pragmatic competence vs. formal-grammatical knowledge, foreign language teaching

\section{Zusammenfassung}

Der vorliegende Beitrag setzt sich zum Ziel, die theoretische Fundierung sowie Vorteile und Schwächen des pragmatisch-kommunikativen Ansatzes im Fremdsprachenunterricht aus glottodidaktiktischer Sicht darzustellen. Die Autorin betont, dass sowohl die Pragmalinguistik und die Sprechakttheorie von J. Austin und J. Searle, die auf theoretischen Erkenntnissen in Bezug auf die Rolle der Handlung und ihrer Wirkung in der pragmatischen Philosophie, Soziologie und Semiotik des 20. Jahrhunderts basieren als auch das Wittgensteinsche Konzept der Sprachspiele, das Konzept der kommunikativen Kompetenz von D. Hymes und die Theorie der kommunikativen Kompetenz von J. Habermas die pragmatisch-kommunikative Wende in entscheidender Weise inspiriert und weitgehend mitgeprägt haben. Die Einführung der kommunikativen Kompetenz in die theoretische Diskussion und in die schulische Praxis zeigte aber nicht immer zufriedenstellende Ergebnisse. Als Schwäche des pragmatischkommunikativen Ansatzes ist eine Überbetonung des kommunikativen Umgangs mit Äußerungen und eine Überbewertung der Rolle der sprachlichen Diskursund Handlungskompetenz bei gleichzeitiger Unterbewertung der morphosyntaktischen Kompetenz zu nennen. Die Autorin konstatiert, dass der immanente Strukturbereich von Sprache und nicht der Kommunikationsbereich ihre Natur ausmacht.

Schlüsselwörter: kommunikativ-pragmatischer Ansatz, Glottodidaktik, Generative Grammatik Sprechakttheorie, kommunikativ-pragmatische Kompetenz vs. formal-grammatisches Wissen, Fremdsprachenunterricht

\section{Problemstellung}

In der zweiten Hälfte des 20. Jahrhunderts ist es $\mathrm{zu}$ einer sprunghaft angestiegenen Popularität des Begriffs „Kommunikation“ gekommen. Diese Popularität wurde entscheidend durch die zunehmende Verbreitung der elektronischen Massenmedien begünstigt. „Kommunikation“ und „kommunikativ“ avancierten zu den zentralen Begriffen, derer man sich in den unterschiedlichen Forschungsdisziplinen bediente. Der berühmte polnische Journalist und Schriftsteller Ryszard Kapuściński (1994: 4) konstatierte: „Wir sind Zeugen - und oft auch aktive Teilnehmer - einer bahnbrechenden Entwicklung, die in ihrer Tragweite mit der Erfindung des Buchdrucks durch Gutenberg im 15. Jahrhundert vergleichbar ist.“ Die Hinwendung der Geistes- 
und Sozialwissenschaften zur Kommunikationstheorie bei gleichzeitiger verbreiteter Problematisierung der Massenkommunikation und ihrer Einflüsse wurde somit als deutliches Merkmal des Zeitgeistes angesehen.

Das Bedürfnis nach einer fundamentalen Neuorientierung führte sowohl in der Linguistik als auch in der Fremdsprachendidaktik (FD) zu einer so genannten „kommunikativ-pragmatischen Wende“, nach der die Funktionen der Sprache im komplexen Gefüge der Kommunikation und der sozialen Interaktionen die zentrale Aufmerksamkeit nach sich zogen. Dabei ging es in der FD vor allem darum, die bisherige Dominanz der Grammatik und die damit verbunden systematische Entwicklung der formal-grammatischen Kompetenz zu überwinden und die Kommunikation, ihre Inhalte und Ziele, d.h. den Aufbau der kommunikativen Kompetenz in den Mittelpunkt des gesamten Fremdsprachenlehr- und -lernprozesses zu stellen. Ein Vorteil eines solchen Versuches sollte darin bestehen, die Kommunikation in den didaktischmethodischen Diskursen im Fremdsprachenunterricht lebensnäher und lebenspraktischer zu gestalten, wobei Lernerzentriertheit, Sprechintentionen, Sprachmittel und ihre Wirkung, mündliche Ausdrucksfähigkeit Authentizität, Alltagssprache und Alltäglichkeit, Handlungsorientierung sowie soziale Sprachspiele als besondere Herausforderung proklamiert wurden.

Wichtig ist $\mathrm{zu}$ betonen, dass im Rahmen der kommunikativpragmatischen Wende in der FD zunächst auf unterschiedliche Theorien und Konzeptionen zurückgegriffen wurde, die mit den „kommunikativen“ und „pragmatischen” in Verbindung gebracht werden konnten, ohne sie auf ihre Kompatibilität hin zu befragen. Die Anhänger der kommunikativen Didaktik hatten sich ausschließlich dem Lernziel „kommunikative Kompetenz“ verschrieben und demnach galt kommunikative bzw. funktionalkommunikative Kompetenz lange Zeit als Leitziel des Fremdsprachenunterrichts, auch wenn seine konkrete Bedeutung zunehmend unklar geworden ist. Es ist unumstritten, dass die Wertschätzung von Lehrund Lernzielen im Fremdsprachenunterricht (FU) zeitbedingt und zeitgebunden ist und dass eine Reihe von Wissenschaften als Bezugsdisziplinen die FD in entscheidender Weise inspiriert und weitgehend mitgeprägt haben. Immanente Interdisziplinarität, perspektivische Umkehr von einer Lehrer- auf eine Lernerbezogenheit sowie Verlagerung des unterrichtlichen Schwerpunktes von einer Formbetrachtung auf einen funktional angemessenen, effizienten Gebrauch der Fremdsprache werden weltweit als eine Voraussetzung für die Eigenständigkeit der kommunikativen Fremdsprachendidaktik anerkannt. 
An dieser Stelle muss darauf hingewiesen werden, dass sich die kommunikative Didaktik in Opposition zu Noam Chomsky positionierte und ihre Begründer und Anhänger vorwiegend in Europa sehr deutlich von dem nativistischen Paradigma der generativen Grammatik und damit von linguistischen Grundpositionen Chomskys distanzierten. Sie haben einen formalen und stark grammatisch orientierten Sprachbegriff verworfen und wandten sich in großem Umfang den Sozialwissenschaften und funktionalistisch-diachronischen Ansätzen in der Linguistik zu, die davon ausgehen, dass die Natur der Sprache in ihrem instrumentellen Charakter als Kommunikationsmittel $\mathrm{zu}$ sehen ist. Besonders inspirierend waren für sie Impulse aus der Sprechakttheorie von John Austin (1962/1972) und John Searle (1969/1971), die sich auf illokutionäre Kräfte konzentrierten, und zwar in dem Sinne, dass Sätze zum Reden und zum Handeln da sind. Da die Sprechakttheorie selbst auf theoretischen Erkenntnissen in Bezug auf die Rolle der Handlung und ihrer Wirkung in der pragmatischen Philosophie, Soziologie und Semiotik des 20. Jahrhunderts basiert, wandte sich dann auch die kommunikativ orientierten Fremdsprachendidaktiker direkt oder indirekt dem Philosophischen Pragmatismus, der Semiotik mit ihrer Beschreibung der syntaktischen, semantischen und pragmatischen Aspekten von Zeichen sowie dem späten Ludwig Wittgensteinschen Konzept „Sprachspiel“ zu. Dabei wurden die ungleichen Zielsetzungen der erkenntnistheoretischen Fundierung der eben genannten Bezugsdisziplinen selbst und der Fremdsprachendidaktik außer Acht gelassen. Unbeachtet blieb die grundsätzliche Verschiedenheit theoretischer und angewandter Wissenschaften. Die Schwierigkeiten des Dialogs zwischen den wissenschaftlichen Diskurswelten werden mit Recht auch heutzutage (vgl. z.B. Ellis/ Shintani (2014: 2ff.) auf die unterschiedlichen „epistemological bases“ zurückgeführt, wobei die Frage nach den relevanten Bezugswissenschaften für die Fremdsprachendidaktik nach wie vor kontrovers diskutiert wird (vgl. Schmenk 2015: 3-16; 2019: 15).

Ohne im Rahmen des vorliegenden Beitrags nur einen annähernden Anspruch auf Vollständigkeit erheben zu können, wird in erster Linie der Versuch unternommen, auf den theoretischen Hintergrund für die kommunikative Fremdsprachendidaktik kritisch einzugehen. Dabei soll gründlich überprüft werden, ob und inwieweit die Forschungsergebnisse der oben genannten Bezugsdisziplinen, die seit den 70er Jahren des 20. Jahrhunderts bis heute mit gewissen Ergänzungen und Veränderungen weltweit geltende kommunikative Didaktik entscheidend beeinflusst haben, eine fremdsprachendidaktische Relevanz in der Wirklichkeit aufweisen und warum das Konzept der kommunikativen Kompetenz in den 
fremdsprachlichen Diskursen im Klassenzimmer in vielen Fällen auf die Trivialität reduziert wurde, d.h. warum die Entwicklung der kommunikativpragmatischen Kompetenz zum Leitziel des Fremdsprachenunterrichts nicht immer zufriedenstellende Ergebnisse in der Schulpraxis zeigte. Des Weiteren wird aus glottodidaktischer Sicht gefragt, ob bzw. inwieweit das Konzept als Ganzes und die kommunikativ-pragmatische Kompetenz als Schlüsselqualifikation im Lichte der neuen Herausforderungen für die Fremdsprachendidaktik im 21. Jahrhundert ausgebaut bzw. aufgegeben werden sollten. Es gilt auch zu hinterfragen, wie der Begriff der kommunikativen Kompetenz im heutigen Fremdsprachenunterricht, d.h. in Zeiten von Interkulturalität, Multimedialität und Digitalisierung neu zu bestimmen ist dies würde jedoch den Rahmen des Artikels bei weitem sprengen.

\section{Kritik an der Generativen Grammatik Chomskys}

Es besteht kein Zweifel daran, dass insbesondere eine von der Linguistik geleistete Beschreibung und Erklärung der Mechanismen der menschlichen Sprachbeherrschung sowie der sie strukturierenden Prinzipien von grundsätzlicher Bedeutung für die Fremdsprachendidaktik sind. Gerade in der Linguistik, die in ihren verschiedenen Heranweisen die menschliche Sprache untersucht, werden sowohl rein spekulativen als auch immer mehr testbare Hypothesen aufgestellt, die es ermöglichen sehr theoriebasiert an zentrale Fragestellungen auch in der Fremdsprachendidaktik heranzugehen.

Die kommunikativ orientierten Fremdsprachendidaktiker haben von Anfang an versucht, sich bewusst von dem Paradigma der generativen Grammatik abzugrenzen, was m.E. teilweise auch negative Konsequenzen hatte. Mit anderen Worten, es war ein Kardinalfehler der kommunikativen Didaktik, die durch Chomsky eingeleitete kognitive Wende in der Linguistik und damit die generativen Prinzipien, die Grundannahmen über die Natur der Sprache sowie ihre rein strukturimmanenten Erklärungsbemühungen aus den Augen verloren zu haben. Das generative Paradigma, das wie kein anderes linguistisches Konzept des 20. Jahrhunderts die Diskussionen auf den Feldern der Sprachphilosophie und der Sprachtheorie weltweit beeinflusst hat, fand jedoch in europäischen fremdsprachendidaktischen Konzeptionen - im Gegensatz zu US-amerikanischen - kaum Eingang. Appel (2017: 87) schreibt dazu: „Das lag einmal daran, dass die generative Grammatik weitaus weniger Anwendungsmöglichkeiten für den Fremdsprachenunterricht bot als der Strukturalismus." Eine solche Meinung ist aber m.E. weitgehend unbegründet. 
In der generativen Linguistik unter dem Einfluss Chomskys, die auch eine prominente aktuelle Forschungsrichtung darstellt, sind gute und zunehmend verbesserte Theorien abstrakt-struktureller Aspekte der menschlichen Sprache erstellt worden (vgl. z.B. Chomsky 1975, 1981, 1986, 1988, 1995, 2002, 2016), die nach wie vor für die Glottodidaktik inspirierend sind. Hier muss nachdrücklich betont werden, dass für N. Chomsky (1975: 29ff.) die menschliche Sprache primär kein allgemeines Kommunikationsmittel ist; im Mittelpunkt von Sprache steht die Syntax, die ausschließlich der menschlichen Spezies eigene Fähigkeit, sprachliche Symbole in bestimmten, spezifizierbaren Folgen immer wieder zu kombinieren, um potenziell unendlich viele grammatisch korrekte Sätze zu erzeugen und zu verstehen. In der Sprache wird von „endlichen Mitteln unendlichen Gebrauch“ gemacht (vgl. Humboldt 1973: 477, Erstauflage 1836) und dies ist ein Wesensmerkmal aller natürlichen, lernbaren Sprachen: „the quintessential property oft he human language“ (Pinker 1997: 118) Auch Fanselow/Felix (1987: 69) als Anhänger der Generativen Grammatik argumentieren auf folgende Art und Weise: „Das, was Sprache ausmacht, ist nicht, dass man mit ihr kommunizieren kann, sondern über welchen Strukturmechanismus man mit ihr kommunizieren kann ". Damit tritt automatisch der Strukturbereich und nicht der Kommunikationsbereich von Sprache in den Vordergrund." (vgl. dazu Sadownik 1997, 2010).

Nach Chomsky ist es absurd anzunehmen, dass Grammatik - der formale Aspekt der Sprache - in irgendeiner Art und Weise aus den pragmatischkommunikativen Bedürfnissen des Menschen resultieren könnte. Anders gesagt, die Annahme, dass sich sämtliche syntaktischen Gesetzmäßigkeiten natürlicher Sprachen aus semantischen und pragmatischen Prinzipien sowie aus kommunikativen Erfordernissen ableiten lassen, ist für die generative Konzeption unakzeptabel. Wenn jemand die Prinzipien, die die Grammatik einer Sprache ausmachen, nicht nur adäquat beschreiben, sondern darüber hinaus erklären wolle, warum sie sind, wie sie sind, stelle, so Chomsky, die biologische Organisation des menschlichen Geistes/Gehirns eine adäquate Begründungsinstanz dar. Die menschliche Sprache wird in der generativen Grammatik als ein Teil der biologischen Ausstattung des Menschen bzw. als Berechnungsmodul des Geistes/Gehirns aufgefasst:

„[...]it seems that we should think of knowledge of language as certain state of mind/brain, a relatively stable element in transitory mental states; furthermore, as a state of some distinguishable faculty of the mind, the language faculty, with its prosperties, structure and organisation, one ,module' of the mind." Chomsky (1986: 5) 
Absurd ist, so auch Chomsky (2011: 263-278), anzunehmen, dass im Bereich des Erwerbs einer kognitiven Struktur wie der Sprache die soziale Umgebung der dominierende Faktor ist, dass Menschen Sprachen ausschließlich dazu benutzen, in einer soziokulturell definierten Umwelt mit anderen Menschen zu kommunizieren. Chomsky setzt sich kritisch mit dem sozialwissenschaftlichen Standardmodell auseinander, nach dem die menschliche Sprache als direktes Resultat von Eigenschaften der gesamten menschlichen Kognition und Bedürfnissen in der Kommunikation zu verstehen ist. Nicht akzeptabel ist für ihn die Annahme, dass sich der Spracherwerb durch allgemeinere Lernprozesse und die Entwicklung sozial-kognitiver Fähigkeiten wie das Etablieren gemeinsamer Aufmerksamkeit, um auf ein Objekt zu referieren, oder die Fähigkeiten, Intentionen der anderen Akteure zu erfassen, erklären lasse, wie z. B. Tomasello (2004: 642ff.) hervorhob.

Laut Chomsky (1986: 209-228, 1995: 145f.) sind Kinder mit einem angeborenen Programm ausgestattet, das den Grammatiken sämtlicher Sprachen gemeinsam ist, d.h. mit einer I(nternalized)Grammatik bzw. Universalgrammatik, die man sich als eine Menge von Prinzipien vorstellen kann, die die Klasse von Sprachen spezifiziert, die vom Kind unter normalen Bedingungen erlernbar sind: „The I-language [...] is some element oft he mind oft he person who knows the language, acquiered by the learner, and usef by the speaker-hearer" (Chomsky 1986: 220). Mit anderen Worten, die Universalgrammatik ist eine Spezifizierung dessen, was ein Kind von Natur aus an kognitiven Strukturen mitbringt, um den Spracherwerb bewältigen zu können, d.h. aus der gesprochenen Sprache ihrer Eltern die syntaktischen Mustern schnell und leicht herauszufiltern. Der Erwerb jeder beliebiger natürlicher Sprache, aber auch weiterer Sprachen wird deshalb nicht als Lernvorgang, sondern als eine Art Reifungsprozess aufgefasst, wobei laut Chomsky (1988: 134) die Sprache als „mentales Organ“ wächst (vgl. Sadownik 2019: 155-168).

Inspiriert von Chomskys Universalgrammatik - die immer wieder z. B. von Tomasello (2004: 642-644, 2009: 470ff., 2011, 2014) aber auch von Trettenbrein (2017) kritisch angegriffen wird - verweist auch Steven Pinker (1998: 341) nicht nur auf die enorme Komplexität der Syntax von Sprache, sondern versucht auch zu beweisen, dass diese Komplexität ihre Berechtigung hat: „Die Feinheiten der Syntax haben in der Geschichte der Psychologie eine herausragende Rolle gespielt, weil sie ein Beispiel für geistige Komplexität sind, die nicht durch Lernen entsteht. Nun - vielmehr wird das Lernen durch die geistige Komplexität ermöglicht. Und das ist wirklich sensationell!“ Dabei 
wird betont, dass die Sprache kein kulturelles Arteffekt ist, das der Mensch auf dieselbe Art und Weise erlernen kann wie beispielsweise das Lesen einer Uhr. Die Sprache ist laut Pinker qualitativ bei allen Menschen gleich und von allgemeinen Fähigkeiten wie dem Verarbeiten von Informationen oder intelligentem Verhalten zu trennen:

„In ihr manifestiert sich auch nicht eine allgemeine Fähigkeit, mit Symbolen umzugehen - wie wir sehen werden, ist ein dreijähriges Kind ein grammatisches Genie, aber völlig unbeschlagen auf dem Gebiet der bildenden Kunst, der religiösen Ikonographie, der Verkehrszeichen und den anderen Bereichen des semiotischen Spektrums.“" (Pinker 1998: 341)

Die generativ orientierte Argumentation war für die meisten Anhänger der pragmatisch-kommunikativen Wende in der Linguistik und in der Fremdsprachendidaktik nicht akzeptabel. Sie griffen vorrangig, wie oben angedeutet, auf die linguistische Pragmatik bzw. die Sprechakttheorie zurück und somit auf ihre erkenntnistheoretische Fundierung, wie die pragmatische Philosophie, Soziologie, Semiotik sowie das Sprachspielkonzept. Die interdisziplinäre Vorgehensweise sollte es ermöglichen, den Lernund Lehrprozess von Fremdsprachen präziser zu analysieren und zu verbessern. Ohne Zweifel wurden dabei neue Kategorien zum Erfassen fremdsprachlichen Unterrichts gewonnen, die diese Bezugsdisziplinen in die Diskussion gebracht und die im Folgenden diskutiert werden.

\section{Der philosophische Pragmatismus - Annahmen und Wirkung}

Den fruchtbaren Boden für die pragmatische Wende in der Linguistik siebziger Jahren des 20. Jahrhunderts hat insbesondere die pragmatische Philosophie des 19. und 20. Jhs. vorbereitet, die in erster Linie die in der Philosophie vorherrschende Trennung zwischen Erkenntnis und Handeln sowie zwischen Theorie und Praxis scharf kritisiert hat. Als bahnbrechend erwiesen sich vor allem die Ideen des amerikanischen Philosophen Charles Sanders Peirce (1839-1914), der inzwischen zum Philosophen des 21. Jahrhunderts ernannt wurde. Interessant ist die Tatsache, dass die Wirkung des sprachphilosophischen Denkens von Peirce, von einigen Ausnahmen abgesehen, erst vor etwa 50 Jahren langsam eingesetzt hat. Obwohl die in den sechziger Jahren einsetzende Beschäftigung mit der systematischen Philosophie das gesamte Werk von Peirce entdeckte und zwar, dass seine Phänomenologie, formale Logik, Metaphysik, Philosophie des Geistes, Semiotik und 
Wissenschaftstheorie systematisch bedeutsame und originelle Vorschläge zu Problemen liefern, die unabhängig von der methodologischen These des Pragmatismus sind (vgl. z.B. Nagl 1998: 34ff., Oehler 1993: 52ff.), wird Peirce auch in der Folgezeit in erster Linie als Begründer des Pragmatismus rezipiert (vgl. Apel 1975: 77ff.).

Nach Peirce ist der Pragmatismus keine Theorie der sprachlichen Bezugnahme, sondern eine semiotisch-logische Methode, die dazu dient, die intellektuelle Bedeutung unseres gegenständlichen Denkens zu klären. Entscheidend für das semiotische Konzept von Peirce ist die Annahme, dass Zeichen niemals isoliert Bedeutung erzeugen können, sondern dass dies ausschließlich unter Rückgriff auf und in Relation zu anderen Zeichen möglich ist. Indem Zeichen wiederholt dieselbe Bedeutung zugeschrieben bekommen, entwickelten sich Konventionen. Mit andern Worten, die Bedeutung einer Aussage wird allein durch die praktischen Konsequenzen der Übernahme dieser Aussage für unser Handeln vorstellbar und konkret entscheidbar, wie es in der Peirce' klassischen Version der pragmatischen Maxime heißt: "Consider what effects that might conceivably have practical bearings you conceive the objects of your conception to have. Then, your conception of those effects is the whole of your conception of the object." (Peirce 1905: 438)/dt. „Überlege, welches die Wirkungen sind, die denkbarerweise praktische Bedeutung haben, durch die wir das Objekt unseres Begriffes denken. Der Begriff dieser Wirkungen ist dann der vollständige Begriff des Objektes“ (übers. von G. Wartenberg 1991: 390). Peirce hat seine pragmatische Maxime in einer Vielzahl von Varianten über einen Zeitraum von dreißig Jahren immer wieder neu formuliert. „Sprachliche Bedeutung“ ist für ihn keine Eigenschaft und keine formale Struktur, die sprachlichen Ausdrucksformen per se zukommt. Bedeutung kann nur extern in Bezug auf jene möglichen Interpretationen und Folgen für unser Handeln erfasst werden, welche die Beziehung zwischen Sprache und Handeln ebenso wie das Sprachund Ausdrucksverhalten des Menschen bestimmen. Hervorzuheben ist in diesem Zusammenhang der Begriff der "Wirkungen“, in denen die Bedeutung der Dinge begründet liegt. Durch die Einschätzung der potenziellen Wirkungen einer Sache wird zugleich der Grad ihrer „Nützlichkeit“ festgelegt (vgl. dazu auch Hookway 2012). Das Kriterium der Nützlichkeit wurde in der Folgezeit von William James (1842-1910) und John Dewey (1859-1952) jedoch überbetont und auf die Gliederung „Wahrheit=Nützlichkeit" reduziert, nach der sich die Wahrheit einer Aussage nur durch ihren praktischen Nutzen beweisen soll. Gegen den Instrumentalismus und Utilitarismus von James und 
Dewey, die er als Verkennung seiner ursprünglichen Ideen empfand, hat Peirce einen neuen Begriff für seinen „Pragmatismus“ etabliert - den „Pragmatizismus“, der „hässlich genug ist, um vor Kinderräubern sicher zu sein“ (Peirce zitiert nach Nagl 1998: 29).

Die Rezeption der semiotischen Schriften ging eigentlich in erster Linie von Charles W. Morris (1938) aus. Von wenigen Ausnahmen abgesehen, beschränkte sich die Kenntnis von Peirce' Semiotik auf einige wenige, immer wieder und meistens bei Morris zitierte Stellen über Ikon, Index und Symbol. Noch heutzutage wird von vielen Autoren Peirce zugeschrieben, was Morris' irreführende These ist, dass nämlich jedes der drei Zeichenkorrelate - Zeichen selbst, Objekt, Interpretant - ausschließlich durch eine der semiotischen Disziplinen Syntax, Semantik und Pragmatik behandelt wird. An dieser Stelle muss nachdrücklich betont werden, dass vorwiegend der Standpunkt von Peirce in der Sprechakttheorie deutlich weiter verfolgt und präzisiert wurde.

Unter dem Einfluss der pragmatischen Philosophie standen insbesondere die Sozialwissenschaften, die u.a. die Rolle der Sprache in der Erzeugung, Aufrechterhaltung und Veränderung der sozialen Wirklichkeit untersuchen. Als richtungweisend erwies sich die „Theorie des symbolischen Interaktionismus“, des amerikanischen Sozialpsychologen Georg Herbert Mead (1863-1931), nach der die Ich-Identität eines Menschen vor allem durch die Teilnahme an interaktiven sozialen Handlungen herausgebildet und entfaltet wird. Die Einflussnahme auf den Anderen erfolgt nach Mead (1934/dt.1973: 22ff.) durch das Medium der Sprache dadurch, dass der Sprecher sich in die Perspektive des Anderen hineinversetzt und aus dieser heraus feststellt, welche Erfahrungen er bei dem Anderen jeweils voraussetzen kann und welche Konsequenzen oder Wirkungen seine Sprechhandlungen bei ihm hervorrufen können. Diesen Mechanismus wird folgendermaßen beschrieben:

„Die vokale Geste einer jener gesellschaftlichen Reize, der das sie gebrauchende Wesen auf die gleiche Weise beeinflusst, wie er es beeinflussen würde, wenn er von einem anderen Wesen käme. Das heißt, dass wir uns selbst sprechen hören können, wobei die Bedeutung des Gesagten für uns die gleiche ist wie für andere." Mead (1934/dt. 1973: 101)

Eine solche Fähigkeit zur Perspektivenübernahme des jeweils anderen gilt es daher zu fördern und zu entwickeln, um eine erfolgreiche Kommunikation zwischen den Gesprächspartnern gewährleisten zu können. Der Gedanke der Perspektivenübernahme des Anderen in der kommunikativen Interaktion wurde später weiter entwickelt in der Gesprächs- und Konversationsanalyse, 
in der interaktionsbezogene Aspekte der Illokutionsmodellierung eine entscheidende Rolle spielen. Meads Postulate einer gleichberechtigten Interaktion zwischen den Gesprächspartnern wurde dagegen später u.a. von Jürgen Habermas (1971) in seiner „Theorie der kommunikativen Kompetenz“ wieder aufgegriffen.

\section{Sprache als Spiel - Ludwig Wittgensteins Sprachspielkonzept}

Als direkt bzw. indirekt wirkende Quelle der Kommunikationstheorie und Pragmalinguistik sowie ihrer Anwendung im Rahmen der Kommunikativen Didaktik ist Ludwig Wittgensteins (1889-1951) pragmatische Fundierung der Bedeutung, insbesondere sein Konzept der Sprachspiele, zu nennen. Es ist wichtig $\mathrm{zu}$ betonen, dass in Wittgensteins späteren Überlegungen eine deutliche Abkehr von seinen früheren Ansichten vollzogen hat. Die Tiefe des Einschnitts wird in der Sekundärliteratur unterschiedlich bewertet. Während die einen sein Schaffen mit Recht in den frühen und den späten Wittgenstein einteilen, versuchen andere Interpretationen, eine Kontinuität in seinem Denken festzuhalten (vgl. dazu u.a. Kienzel 2007).

Die „Philosophischen Untersuchungen“ (PU) werden gerne als Paradigmenwechsel in der analytischen Philosophie gedeutet. Wittgenstein hat in seinem Spätwerk eine völlig neue Vorstellung davon ausgearbeitet, wie der Spracherwerb beim Kind prinzipiell abläuft und was das Wesen der Sprache ist. Dazu benötigte er eine Reihe neuer Begriffe, die er nach und nach einführte und erläuterte. Wittgenstein bemühte sich hier nicht mehr um die Klärung der Frage, wie die Sprache auf die Wirklichkeit bezogen ist. Viel wichtiger war jetzt für ihn die Reflexion über den Gebrauch der Sprache im Allgemeinen. Auf das Gebrauchsmodell der Sprache kommt Wittgenstein im Zuge seiner Überlegungen, wie eigentlich die Bedeutungen von sprachlichen Ausdrücken gelernt werden. Er geht davon aus, dass sprachliche Ausdrücke ihre Bedeutung durch ihren Gebrauch bekommen. Um die Bedeutung von Wörtern zu erfassen, sollte man, so (Wittgenstein 1984: 262, Bd.1, § 43, Erstauflage 1953) ihre verschiedenen Funktionen untersuchen: „Die Bedeutung eines Wortes ist sein Gebrauch in der Sprache. Und die Bedeutung eines Namens erklärt man manchmal dadurch, daß man auf seinen Träger zeigt." Nach Wittgenstein muss man den allgemeinen Gebrauch des Wortes beherrschen, um ein Wort zu verstehen. Der Begriff der „Bedeutung“ wird in den PU deutlich durch den Begriff des „Gebrauchs“ 
ersetzt. Durch die Akzentuierung auf den Gebrauch der Sprache, d.h. durch die Rückführung eines Ausdrucks auf dessen Gebrauch, ergab sich u.a. die Frage, wodurch die Korrektheit festgelegt ist. Sprachliche Ausdrücke setzen Regeln voraus. Solche Regeln können nach Wittgenstein nicht als starre Vorschriften der Verwendungsweise verstanden werden, vielmehr drücken sie analog den Satzmodi verschiedene Arten der Verwendung von Sätzen aus. Wittgenstein führte dazu den neuen Begriff des "Sprachspiels“ ein, der in seinem Werk einen zentralen Platz einnimmt: „Ich werde auch das Ganze: der Sprache und der Tätigkeiten, mit denen sie verwoben ist, das "Sprachspiel« nennen (1984: 54, Bd.1, §7) Er verweist auf die Analogie zwischen Sprache und Spiel. Anhand des Sprachspielbegriffes wird seine Auffassung von Sprache erläutert, und zwar, dass zum Verständnis einer Sprachverwendung die gesprochenen Wörter allein nicht ausreichen, sondern dass darüber hinaus auch die typischen Tätigkeiten, die das Sprechen begleiten, zum Sprachgebrauch gezählt werden müssten. „Deswegen werde hier ein neuer Begriff eingeführt, der diesem erweiterten Blick durch die Verknüpfung der Worte „Sprache“ und „Spiel“ Rechnung trägt“ (zit. nach Kienzler 2007: 27). Folgend Wittgenstein können die verschiedenen Satzmodi als Ausdruck verschiedener Sprachspiele verstanden werden. Er nennt "Sprachspiele“ die mannigfachen Weisen, auf die Worte und Sätze in der Sprache verwendet werden: „Das Wort Sprachspiel soll hier hervorheben, dass das Sprechen der Sprache ein Teil ist einer Tätigkeit oder einer Lebensform" (Wittgenstein 1984: 150, Bd.1, § 23). Infolge einer Auseinandersetzung mit einem möglichen Mangel seines Sprachspielkonzeptes führte Wittgenstein mit „Lebensform“ einen zweiten neuen Begriff ein (Wittgenstein 1984: 88ff., Bd.1 §19 bis §22). Das Interessante an Wittgensteins „Sprachspiel“ ist seine Verknüpfung zwischen dem Tätigsein und dem Sprechen. Dadurch verwurzelt Wittgenstein die Sprache als Sprechen mitten im Leben, das aus vielen gemeinsamen einfachen Reaktionen, komplexen Verhaltensweisen und Handlungen einer Sprechgemeinschaft besteht: „Der Ursprung und die primitive Form des Sprachspiels ist eine Reaktion; erst auf dieser können die komplizierteren Formen wachsen. Die Sprache - will ich sagen - ist eine Verfeinerung, im Anfang war die Tat'." (Wittgenstein 1984: 493, Bd.8, §545).

Ein Sprachspiel besteht aus einer Folge von sprachlichen Äußerungen in einer bestimmten äußeren Situation und in einem bestimmten Handlungskontext. So ist auch das Benennen der Dinge als ein kompliziertes Sprachspiel anzusehen, das nur von dem verstanden werden kann, der bereits die Technik des Gebrauchs der Sprache erlernt hat. Der Gebrauch der Sprache kann also nicht außerhalb eines Sprachspiels erlernt werden, da Lernen den 
Gebrauch im richtigen Kontext voraussetzt. Mit anderen Worten, das Sprechen einer Sprache ist für Wittgenstein wie ein Spiel, eine von Regelmäßigkeiten, von wiederholten Spielhandlungen in der Zeit durchsetzte, wenn auch nicht in jeder Hinsicht von Regeln begrenzte Tätigkeit des Operierens mit Worten. Sie dient keineswegs allein dem Ausdruck oder der Mitteilung von Gedanken, sondern verfolgt, abhängig von der Lebensform der Menschen, der sie entspringt, ganz unterschiedliche Zwecke, was deutlich wird, wenn man sich, so Wittgenstein, die mannigfachen Arten von Sprachspielen, die wir im Leben wirklich benutzen, vor Augen führt, wie z.B. einen Erlebnisbericht geben, ein Märchen erzählen, eine Einrichtung beschreiben, einen Befehl erteilen, eine Frage stellen, Bitten, Danken, Fluchen, Grüßen, Beten und andere mehr: „Man kann sich leicht eine Sprache vorstellen, die nur aus Befehlen und Meldungen in der Schlacht besteht. - Oder eine Sprache, die nur aus Fragen besteht und einem Ausdruck der Bejahung und der Verneinung. Und unzählige Andere. - Und eine Sprache sich vorstellen heißt, sich eine Lebensform vorstellen (Wittgenstein 1984: 215, Bd. 1, § 19). Des Weiteren weist Wittgenstein (1984: 250, Bd. 1, § 23) auf die Vielzahl von sprachlichen Verwendungsmöglichkeiten hin:

„Wieviele Arten der Sätze gibt es aber? Etwa Behauptung, Frage oder Befehl?Es gibt unzählige solcher Arten unzählige verschiedene Arten der Verwendung alles dessen, was wir "Zeichen«, »Worte«, "Sätze« nennen. Und diese Mannigfaltigkeit ist nichts Festes, ein für allemal Gegebenes; sondern neue Typen der Sprache, neue Sprachspiele, wie wir sagen können, entstehen und andre veralten und werden vergessen.[...] Führe dir die Mannigfaltigkeit der Sprachspiele an diesen Beispielen vor Augen:

Befehlen und nach Befehlen handeln -

Beschreiben eines Gegenstandes nach dem Ansehen, oder nach Messungen -

Herstellen eines Gegenstandes nach einer Beschreibung (Zeichnung) -

Berichten eines Hergangs -

Über den Hergang Vermutungen anstellen -

Eine Hypothese aufstellen und prüfen -

Darstellen der Ergebnisse eines Experiments durch Tabellen und Diagramme -

Eine Geschichte erfinden; und lesen -

Theater spielen -

Lieder singen -

Rätsel raten -

Einen Witz machen, erzählen -

Ein angewandtes Rechenexempel lösen -

Aus einer Sprache in die andere übersetzen -

Bitten, Danken, Fluchen, Grüßen, Beten.“ 
Sprechen und Spielen sind laut Wittgenstein miteinander verwandt, und zwar in der Hinsicht, dass beide Teil einer Lebensform sind. Eine Lebensform umfasst also sowohl die Spiele als auch die Sprachspiele und vieles, vieles mehr. Anhand der Verwandtschaftsverhältnisse der Sprachspiele führte Wittgenstein seinen dritten neuen Begriff ein: „Verwandtschaft“ bzw. „Familienähnlichkeit“ ein. Er schreibt dazu:

„Hier stoßen wir auf die große Frage, die hinter allen diesen Betrachtungen steht. - Denn man könnte mir einwenden: „Du machst dir's leicht! Du redest von allen möglichen Sprachspielen, hast aber nirgends gesagt, was das Wesentliche des Sprachspiels, und also der Sprache, ist. Was allen diesen Vorgängen gemeinsam ist und sie zur Sprache, oder zu Teilen der Sprache macht. [...]" Und das ist wahr. - Statt etwas anzugeben, was allem, was wir Sprache nennen, gemeinsam ist, sage ich, es ist diesen Erscheinungen gar nicht Eines gemeinsam, weswegen wir für alle das gleiche Wort verwenden, - sondern sie sind miteinander in vielen verschiedenen Weisen verwandt. Und dieser Verwandtschaft, oder dieser Verwandtschaften wegen nennen wir sie alle „Sprachspiele“. Ich will versuchen, dies zu erklären.“ (Wittgenstein 1984: 331, Bd. 1, § 65)

Worte gewinnen nur in bestimmten Sprachspielen, d.h. in einer gewissen Umgebung oder vor einem bestimmten Hintergrund des Lebens oder Sprechens ihre Bedeutung: „Frage dich in dieser Schwierigkeit immer. Wie haben wir denn die Bedeutung der Wortes (»gut« z.B.) gelernt? An was für Beispielen, in welchen Sprachspielen?“ (Wittgenstein 1984: 432, Bd. 1, $\S 77)$. Die Sprachkenntnis ist nach Wittgenstein als eine Handlungskompetenz zu charakterisieren, als eine Fähigkeit also, etwas Bestimmtes zu tun. Kommunikation ist demnach eine wichtige Funktion von Sprache und die Erklärung dieser Funktion hat auf ein soziales Konzept von Lebensform zu rekurrieren.

Durch die Gebrauchstheorie der Bedeutung wurde die Eigenständigkeit der tatsächlich gesprochenen Sprache auf besondere Weise herausgestellt. Die Funktion eines Ausdrucks wird nicht dadurch erklärt, dass diesem eine sprachunabhängige und ohne Vermittlung der Sprache erfassbare Entität zugeordnet wird. Die Idee, dass es ein Wesen der Sprache gäbe, das allen sprachlichen Erscheinungen gemeinsam wäre, gibt Wittgenstein also demonstrativ auf. Stattdessen seien diese Erscheinungen miteinander verwandt: „Statt etwas anzugeben, was allem, was wir Sprache nennen, gemeinsam ist, sage ich, es ist diesen Erscheinungen gar nicht Eines gemeinsam, weswegen wir für alle das gleiche Wort verwenden, - sondern sie sind miteinander in vielen verschiedenen Weisen verwandt. Und dieser Verwandtschaft, oder dieser 
Verwandtschaften wegen nennen wir sie alle »Sprachen«. Ich will versuchen, dies zu erklären." (Wittgenstein 1984:432, Bd. 1, § 65b). Die eigenständige Leistung der Sprache zeigt sich gerade darin, dass Ausdrücke erst durch ihre spezifische Verwendungsweise im Alltag etwas bedeuten. Wittgensteins Identifizierung von Gebrauch und Bedeutung sowie seine Ausführungen zum Spracherwerb, wonach die Sprache als eine Form sozialen Lebens mithilfe von Sprachspielen gelernt wird und Sprachkenntnis durch Abrichtung auf solche Sprachspiele vermittelt wird, wurden und werden immer noch weltweit intensiv und kontrovers diskutiert.

Chomsky (1975) hielt Wittgensteins spätes Werk für einen unfundierten und überdies dogmatischen Empirismus. Chomskys Kommentar zu Wittgensteins Konzeption des Sprachenlernens lautet, dass sie in keiner Weise zeigt, und dass es unwahrscheinlich ist, dass sie es je zeigen kann, „wie normale Sprachkenntnis, d.h. die Art von Wissen, die uns das Verstehen irgendeines neuen Satzes ermöglicht, mit Hilfe der Mechanismen beschrieben werden kann, die sich in den eigenartigen Beispielen von paradigmatisch eingeführten „Sprachspielen“ manifestieren und die den Kern der Sprache bilden“ (Chomsky 1975: 339). Chomsky vertrat deutlich die Auffassung, dass sein das Sprachsystem erklärendes Unternehmen mit dem Ansatz Wittgensteins unverträglich sei, da er selbst Sprache als eine Form biologischen Lebens verstand. Grewendorf versucht dagegen zu begründen, entgegen N. Chomskys eigener Auffassung, dass die beiden Unternehmen miteinander verträglich sind:

„Zunächst ist festzustellen, dass Wittgenstein sich nicht mit den Bedingungen für die Fähigkeit, wohlgeformte, strukturierte Sätze zu bilden, befasst hat. Einige paradigmatisch angeführte Sprachspiele, in die mittels $>$ Abrichtung $<$ eingeübt werde soll, betreffen den Gebrauch von Ausdrücken zum Vollzug sprachlicher Handlungen (lokutionärer und illokutionärer Akte im Sinne Austins (1962); sie betreffen also die pragmatische Ebene, auf der das $>$ Wissen $<$ gebraucht wird, für dessen Erwerb Chomsky eine explanative Theorie liefern“. (...) „Da Wittgenstein also in keiner Weise die kausalen Fragen berührt, die Chomsky zufolge eine Theorie des Spracherwerbs zu beantworten hat, kann ihm lediglich Ignoranz, Langweile oder mangelnde Kompetenz - kurz: der Verzicht auf Erklärungen - vorgeworfen werden. (..) Chomsky fühlt sich daher in seinem Unternehmen zu Unrecht von Wittgenstein attackiert." (Grewendorf 1995: 79-84)

Die Überlegungen Wittgensteins in den PU haben in der weiteren Folge dazu geführt, das Sprechen als Handlung als Ausgangslage von Bedeutungsanalysen $\mathrm{zu}$ nehmen. Insbesondere mit der Auffassung, dass 
die Bedeutung an den Gebrauch der Sprache rückgebunden ist, hat er der Sichtweise, Sprechen als eine Form des Handelns zu begreifen, den Weg bereitet. Es war Austin vorbehalten, durch systematisches Studium der normalen Sprache der Sprachpragmatik einen zentralen Stellenwert einzuräumen. Austin kommt das Verdienst zu, die Relevanz von Wittgensteins Andeutungen zum Satzmodus und seinen Ausführungen zur Gebrauchstheorie der Bedeutung erkannt und in spezifischer Weise weiterentwickelt zu haben. Das Sprachspielkonzept hat dagegen die Grundannahmen der kommunikativen Didaktik auf den Ebenen von der Lernzielbestimmung bis hin zu konkreten methodischen Vorschlägen, z.B. ,spielend lernen` weitgehend beeinflusst.

\section{Sprache als eine spezifische Handlungsform. Die Sprechakttheorie von Austin und Searle}

Als Begründer der Sprechakttheorie gelten der englische Moralphilosoph Austin und der amerikanische Sprachphilosoph Searle, der Austins Theorie weiterentwickelt hat. Austin studierte in Oxford zunächst klassische Philologie, bevor er sich mit Philosophie eingehender befasste. Sein Ansatz "Other Minds“ (1946) begründete seine Bedeutung als Repräsentant der Normalsprachphilosophie. Er lehrte an der Universität in Oxford Philosophie. Seine Schrift „How to do things with words“ (1962; dt. „Zur Theorie der Sprechakte“, 1972) und „Sense and Sensibilia“ (1962; dt. „Sinn und Sinneserfahrung“, 1975) sind erst postum veröffentlicht worden. Angeregt durch das Sprachspiel-Konzept des österreichischen Philosophen Wittgenstein fasste Austin die menschliche Sprache als eine spezifische Handlungsform auf. Er richtete seine Kritik ganz deutlich gegen die Postulate der analytischen Philosophie, wonach die Grundlage der philosophischen Erörterungen ihre logischen Analysen bilden sollten, um etwaige Mehrdeutigkeiten, Missverständnisse und Widersprüche eliminieren zu können.

Austin wiederholte Kritik Wittgensteins an der Idealsprache, dass sie kein angemessenes Modell für die tatsächliche Sprache darstellt. In seinen Erörterungen über die Natur der Sprache bemerkte er, dass das Hauptinteresse der (Sprach)philosophen vor allem sprachlichen Aussagen galt, die lediglich nach ihrem Wahrheitsgehalt untersucht wurden, also danach, ob sie falsch oder wahr sind. Dieses Unterscheidungskriterium betrifft nach Austin (1962/dt.1975: 27) nur eine eingeschränkte Gruppe von Aussagen, die etwas beschreiben (deskriptiv) oder etwas behaupten (konstativ). Es gibt jedoch viele Äußerungen, die weder falsch noch wahr sind, die weder als deskriptiv noch 
als konstativ bezeichnet werden können; Austin nannte sie „performative“ Äußerungen, die auf ihr Gelingen bzw. Misslingen begutachtet werden: „Der Name stammt von ,to perform', vollziehen: man vollzieht Handlungen. Es soll andeuten, dass jemand, der solche Äußerung tut, damit eine Handlung vollzieht - man fasst die Äußerung nicht gewöhnlich als bloßes Sagen auf “ (Austin 1962/dt.1975: 28f.). Austin (1962) konzentrierte seine Überlegungen auf die Charakterisierung der Sprechhandlungen, durch die wir Äußerungen tätigen, d.h. er analysierte sprachliche Äußerungen darauf hin, ob und inwiefern sie zugleich Handlungen sind. Dieser handlungstheoretische Ansatz, der in der Folge zum Begriff des „Sprechaktes“ als einer Form menschlichen Handelns führte, bildete eine wesentliche Grundlage für die Etablierung der „Sprechakttheorie“ als Beschreibungskonzept. Er unterscheidet dabei drei Aspekte einer sprachlichen Äußerung:

1. Mit jeder Äußerung wird etwas ausgesagt;

2. Wir können eine Handlung vollziehen, indem wir etwas sagen;

3. Wir können durch den Vollzug einer Handlung, indem wir etwas sagen, eine bestimmte Wirkung erzielen.

Abschließend zeigte Austin, dass die Unterscheidung von perfomativ und konstativ nicht akzeptabel und somit der Gegensatz perfomativ-konstativ aufzugeben ist, auch die Konstative sind Handlungen.

Searle hat die Idee einer Sprechakttheorie weiterentwickelt und wirkungsvoll propagiert („Speech Acts“ 1969 dt. „Sprechakte“ 1971). Was Searle in seiner Studienzeit in den fünfziger Jahren in Oxford insbesondere von Austin gelernt hat, fand demnach eine originelle und maßstäblich gewordene Fortsetzung. Auch Searle analysierte Sprachakte als die kleinsten fundamentalen Einheiten der sprachlichen Kommunikation, verstand Sprechen als regelgeleitetes intentionales Handeln und fasste dementsprechend Sprachtheorie als Teil einer umfassenden Handlungstheorie auf. Searl (1971: 19ff.) schlug aber folgende Strukturierung des Sprechaktes vor:

(1) Äußerungsakt (Lokution) umfasst die Äußerung von Wörtern, Morphemen und Sätzen,

(2) Der propositionale Akt umfasst die unvollständigen, d.h. nur im Zusammenhang mit illokutionären Akten vollziehbaren Sprechakte der Referenz (Bezugnahme auf Objekte der außersprachlichen Welt) und Prädikation (Aussage über Welt/Zusprechen von Eigenschaften),

(3) der illokutionäre Akt zeigt an, in welchem Sinne man eine Äußerung macht, bzw. die Äußerung aufgefasst werden soll,

(4) der perlokutive Akt - intendierte Wirkung des Sprechaktes. 
Der illokutionäre Akt zeigt also an, in welcher Intention die betreffende Äußerung gemacht wird und in welcher Bedeutung die Äußerung zu verstehen ist. Er gibt daher nicht zwei prinzipiell verschiedene semantische Untersuchungen, nämlich eine Untersuchung der Bedeutung von Sätzen und eine des Vollzugs von Sprechakten. Der Sprechakt, der mit der Äußerung eines Satzes vollzogen wird, bildet allgemein eine Funktion der Bedeutung des Satzes. Denn jeder Satz, der Bedeutung hat, kann aufgrund seiner Bedeutung verwendet werden, um einen bestimmten Sprechakt zu vollziehen. Ebenso ist jeder mögliche Sprechakt im Prinzip exakt als Satz formuliert (vgl. Searle 1971: 32ff.). Mit seinen Ausführungen wollte Searle insgesamt zeigen, inwiefern die Grundeinheit der sprachlichen Kommunikation nicht das Symbol, das Wort oder der Satz ist, sondern die Produktion oder Hervorbringung des Wortes oder des Satzes im Vollzug des Sprechaktes: „Genauer ist die Produktion des Satzes unter bestimmten Bedingungen der illokutionäre Akt, und der illokutionäre Akt ist die minimale Einheit sprachlicher Kommunikation“ (Searle 1971: 44). Besonders einflussreich war seine Taxonomie von Sprechakten (Searle 1979/dt. 1982: 33ff), die sich vor allem auf die Kriterien (a) illokutionäre Absicht, (b) psychische Grundlage und (c) Anpassungsrichtung zwischen Sprache und Welt stützt. Die Sprechakttheorie ist durch ihre zentralen Begriffen wie ,Sprechabsichten' bzw. ,Sprechintentionen', 'Notionen' bzw. ,Verständigungsbereiche', sprachliche Mittel' und ihre ,Wirkung' sowie ,Handlungsbezogenheit' ist Gemeingut der fremdsprachendidaktischen Welt geworden; sie steht beispielsweise hinter dem Gemeinsamen Europäischen Referenzrahmen.

\section{Das Konzept der kommunikativen Kompetenz}

Obwohl Austin und Searle die pragmatische Analyse und Beschreibung der menschlichen Sprache begründeten und ausarbeiteten und damit maßgeblich zur Perspektivenerweiterung innerhalb der Linguistik und Soziolinguistik beitrugen, unternahmen sie eigentlich keinen Versuch, kommunikative Kompetenz zu ergründen und zu beschreiben. Diese Aufgabe, kommunikative Kompetenz $\mathrm{zu}$ konzeptualisieren, stellten sich v.a. der amerikanische Anthropologe und Soziolinguist Dell Hymes und der deutsche Soziologe und Philosoph Jürgen Habermas, die pauschal auch als Ursprungsquellen der kommunikativen Didaktik bezeichnet wurden. Eine genaue Analyse der Positionen von Hymes und Habermas zeigt jedoch deutlich, dass beide Wissenschaftler von unterschiedlichen Dimensionen von 
Kommunikation sprechen, wenn sie kommunikative Kompetenz konzeptualisieren.

In seinem Aufsatz „On communicatve competence“ (1972: 269-293) wendet sich Hymes gegen den linguistischen Kompetenzbegriff von Chomsky und kritisiert auch scharf den der generativen Grammatik zugrunde liegenden Sprachbegriff. Ein Grundgedanke seines Begriffes der kommunikativen Kompetenz bestand darin, dass die Beherrschung einer Sprache nicht nur in Beherrschung von Regeln zur Produktion korrekter Sätze bestehe, sondern auch in der Fähigkeit zum Sprachgebrauch. Kompetenz im Sinne Chomskys ist dagegen das sprachliche „Wissen“, über das ein Sprecher implizit verfügt und das die Voraussetzung für den Gebrauch von Sprache bildet. Entscheidend ist bei diesem zentralen Begriff der generativen Theorie, dass es sich dabei um einen Typus von Wissen handelt, das einerseits nicht dem Bewusstsein zugänglich ist und andererseits auch nicht die individuelle Fähigkeit zur Explikation einschließt, d.h. derjenige, der über dieses Wissen verfügt, kann in der Regel nicht angeben, worin denn dieses Wissen konkret besteht. Anders gesagt, der Sprecher kann über dieses Wissen nur in den seltensten Fällen explizit Rechenschaft ablegen. Aus diesem Grund wird dieser Typ von Wissen zumeist als „intuitive knowledge“ oder „tacit knowledge“ bezeichnet (vgl. Chomsky 1975: 15ff.). Gegen diesen Wissensbegriff ist eingewandt worden, dass er im Gegensatz dazu steht, was man üblicherweise unter Wissen versteht. Wissen, so wird argumentiert (vgl. z.B. Searle 1980: 37-38, Baker/ Hacker 1984: 32ff.), impliziert quasi per definitionem, dass sein Inhalt zumindest prinzipiell bewusst gemacht und von demjenigen, der über dieses Wissen verfügt, erklärt werden kann. Die gängige Vorstellung von Wissen schließt demnach typischerweise die explizite Erklärbarkeit des Wissensinhaltes ausdrücklich mit ein.

Hymes beschäftigte sich mit Fragen zu Bedingungen und Möglichkeiten von realer Kommunikation zwischen Menschen (hier speziell von Kindern in unterschiedlichen sozialen und kulturellen Milieus) und versuchte ihre Dynamik und Regularitäten zu erfassen. Kommunikative Kompetenz umfasst Hymes zufolge sowohl Aspekte dessen, was Chomsky als grammatische Kompetenz bezeichnet hatte als auch die Dimensionen des tatsächlichen Sprachgebrauchs. Die kommunikative Kompetenz wird als eine relative, offene Größe aufgefasst, die man erfolgreich erlernen kann. Anders gesagt, die kommunikative Kompetenz wird von Hymes als die Fähigkeit eines Sprechers, über das Was, Wo, Wie und Wann seines Sprechens $\mathrm{zu}$ entscheiden, und damit Sprechen und Verstehen als sinnvolle Aktivität 
in einer Gesellschaft möglich zu machen. Entscheidend für das Verständnis des theoretischen Status dieses Begriffs von kommunikativer Kompetenz ist, dass Hymes kein System oder Theoriekonzept entwerfen wollte, sondern zunächst nur Fragen stellte. Diese Fragen nennt er „sinnvoll“ in Bezug auf die Möglichkeit, Einsichten in die Organisationsprinzipien des Sprechens zu gewinnen. Damit gibt er zu bedenken, dass es ein Zusammenspiel verschiedener Dimensionen von Kommunikationswissen und -können ist, das Menschen kommunikativ kompetent macht. An dieser Stelle muss erwähnt werden, dass die kommunikativ orientierten Didaktiker trotz aller Abgrenzungen von der generativen Grammatik eine an Chomsky angelehnte Terminologie, wie z.B. eine „communicative performance“ (Breen/Candlin 1980: 92), oder eine Kompetenz-Performanz-Unterscheidung (Auer 1999: 197) relativ oft verwendeten und einem ähnlichen Universalismus wie die generative Grammatik folgten. Fundamental waren sie jedoch durch den Rückgriff auf die Sozialwissenschaften geprägt und bezogen sich im Wesentlichen auf die Arbeiten von Habermas, der noch heute als der soziologische ,Großtheoretiker' nicht nur in der Bundesrepublik gilt.

Habermas versuchte hingegen in seinem Beitrag „Vorüberlegungen zu einer Theorie der kommunikativen Kompetenz" (1971) eine Universalpragmatik bzw. eine universale Pragmatik der Kommunikation zu entwickeln, die es ermöglicht, Bedingungen für eine ideale Sprechsituation zu bestimmen, die frei von allen äußeren und inneren Zwängen ist und eine gleichberechtigte Kritik von Meinungen ermöglicht:

„Die ideale Sprechsituation schließt systematische Verzerrung der Kommunikation aus. Nur dann herrscht ausschließlich der zwanglose Zwang des besseren Argumentes, der die methodische Überprüfung von Behauptungen sachverständig zum Zuge kommen läßt und die Entscheidung praktischer Fragen rational motivieren kann“. Habermas (1971: 137)

Die Kommunikation zwischen Menschen sollte laut Habermas idealerweise so verlaufen, dass sie nicht durch Indoktrination, durch hierarchische Strukturen oder durch Ideologien eingeschränkt wird. Ideale Sprechsituationen müssen herrschaftsfrei sein, um zu einem wahren Konsensus zu führen. Ein wahrer Konsensus kann erst dann entstehen, wenn alle Beteiligten als gleichberechtigte Partner am Diskurs teilnehmen. Voraussetzung dafür ist eine „symmetrische Verteilung der Chancen, Sprechakte zu wählen und auszuüben“ (Habermas 1971: 137). Mit seinem Interesse an den Bedingungen der Möglichkeit, einen gesellschaftlichen Konsensus zu erziehen, greift Habermas die philosophische Frage nach dem 
Status der Wahrheit auf und stellt eine Antwort zur Diskussion, die Wahrheit nicht in den Dingen, sondern in den Kommunikationsprozessen von sozialen Interaktanten sucht. Die sog. Konsensustheorie der Wahrheit von Habermas besagt, dass genau diejenigen Ausdrücke wahr sind, über die sich in einer idealen Sprachsituation argumentativ ein Konsens erzielen läßt. Diese Auffassung stellt Habermas allen Versuchen gegenüber, Wahrheit als Übereinstimmung der Erkenntnis mit ihrem Gegenstand zu bestimmen.

Hervorzuheben ist, dass auch der Begriff ,Diskurs' der im Alltagsgebrauch oft mit der Bedeutung ,öffentliches Gespräch oder Debatte über ein bestimmtes Thema' in Verbindung gebracht wird, maßgeblich auf die Theorie kommunikativen Handelns von Habermas (1988b [1981]) zurückgeht. In seiner Kommunikations- und Gesellschaftstheorie unterscheidet er zwischen ,Kommunikation' und ,Diskurs'. Kommunikatives Handeln stellt nach Habermas gewissermaßen den Normalfall des Sprachgebrauchs dar, wenn es also darum geht, das Einverständnis (,Konsens') über etwas herzustellen oder zu erneuern (vgl. 1981: 38ff.). Um einen ,Diskurs' handle es sich hingegen dann, wenn „die Kommunikation gestört ist und kein Einverständnis hergestellt werden kann" (1981: 38) und daher die Grundlagen der Kommunikation selbst thematisiert und argumentativ geklärt werden müssen. Diskurse sind in einer von Habermas (1984: 500) selbst gewählten Metapher „Inseln im Meer der Praxis“ alltäglicher Kommunikation. Zusammenfassend lässt sich festhalten, dass sowohl Habermas' Kommunikations- als auch sein Diskursbegriff prinzipiell konsensorientiert sind: Konsens ist das Ziel von Kommunikation und, sollte diese scheitern, das Ziel von Diskursen. Mit anderen Worten: Diskurse werden geführt, um den reibungslosen Ablauf von Kommunikation wieder herzustellen. Dabei spielt Vernunft eine zentrale Rolle, denn am Ende setzt sich nach Habrmas (Hervorhebung im Original) das „bessere Argument“ kraft seiner Rationalität durch:

„Die Konsensustheorie der Wahrheit beansprucht, den eigentümlich zwanglosen Zwang des besseren Argumentes durch formale Eigenschaften des Diskurses zu erklären und nicht durch etwas, das entweder, wie die logische Konsistenz von Sätzen, dem Argumentationszusammenhang zugrunde liegt oder, wie die Evidenz von Erfahrungen, von außen gleichsam in die Argumentation eindringt. Der Ausgang eines Diskurses kann weder durch logischen noch durch empirischen Zwang allein entschieden werden, sondern durch die ,Kraft des besseren Argumentes.“ (Habermas 1984: 161.)

Habermas geht vom idealen Diskursverlauf aus. Ihn interessieren die Regeln, die zur Realisierung solcher Diskurse führen. Diese Regeln sind es, die die 
pragmatische bzw. kommunikative Kompetenz ausmachen: d.h. (1) gleiche Chancen auf Dialoginitiation und -beteiligung, (2) gleiche Chancen der Deutungs- und Argumentationsqualität, (3) Herrschaftsfreiheit, sowie (4) keine Täuschung der Sprechintention. Diese transzendental pragmatischen Bedingungen ermöglichen Verständigung und einen vernünftigen Diskurs. Habermas verweist darauf, dass diese Idealisierung vor jedem Sprechakt vorgenommen werden sollte. Aus dieser kommunikativen Vernunft und Organisation von Handlungen heraus ergibt sich dann kommunikatives Handeln. Habermas spricht von pragmatischen Universalien, das heißt von einem Regelapparat, der grundsätzlich in Diskursen in jedem sozialen und kulturellen Kontext gültig ist. Sein Kompetenzbegriff stellt eine in Interaktanten idealiter befindliche Größe dar, die sich auf deren grundsätzliche Befähigung zur Teilnahme am Diskurs bezieht. Anders gesagt, Kommunikative Kompetenz ist ein abstrakt gedachtes Potenzial, d.h. etwas, das jeder Mensch potenziell besitzt, unabhängig von persönlichen und sozialen Eingebundenheiten und kulturellen Kontexten. Problematisch erscheinen ihm vor allem die Zwänge kultureller und traditionsbedingter Natur:

„Je mehr kulturelle Traditionen eine Vorentscheidung darüber treffen, welche Geltungsansprüche wann, wo, für was, von wem, und wem gegenüber akzeptiert werden müssen, um so weniger haben die Beteiligten selbst die Möglichkeit, die potenziellen Gründe, auf die sie ihre Ja/Nein-Stellungnahmen stützen, explizit zu machen und zu prüfen." (Habermas 1995: 108).

Mit einer solchen sozialkritisch fundierten Universalpragmatik und der auf ihr aufbauenden kommunikativen Kompetenz will Habermas nicht konkrete sprachtheoretische Probleme lösen, sondern den jeweiligen status quo einer Gesellschaft in Frage stellen und dadurch Veränderungen innerhalb dieser Gesellschaft initiieren, wobei die vererbten kulturbildenden Faktoren den freien Diskurs nur unnötig stören und daher ebenfalls einer rationalen Kritik und Korrektur unterzogen werden müssen.

In der Fachliteratur wird Habermas gegenüber oft der Vorwurf der „Kulturblindheit“ universalistisch gedachter Wahrheitsfindungsprozesse erhoben, man hat ihm die „Realitätsferne“ seines Ideals vom wahren Konsensus vorgeworfen. Interessant ist auch eine heftige Auseinandersetzung mit Habermas als Ideengeber für das Konzept der kommunikativen Kompetenz. Kommunikative Kompetenz beinhaltet Habermas zufolge die Fähigkeit zwischen Sein und Schein, zwischen Sein und Sollen sowie zwischen Wesen und Erscheinung zu unterscheiden. Diese Unterscheidungen verhelfen nach Habermas dazu, zwischen dem wahren (wirklichen) und den falschen 
(täuschenden) Konsensus unterscheiden zu können. Für Portmann (1981: 54) ist die von Habermas entwickelte kommunikative Kompetenz „die Chiffre, dank der es gelingt, einen ursprünglich politischen Begriff der Kritik und Emanzipation sozusagen als strukturellen Faktor in den Begriff der Kommunikation zu integrieren“.

Insgesamt hatte Habermas' Konzeption der Schaffung der idealen Sprechsituation und des herrschaftsfreien Diskurses einen großen Einfluss auf gesellschaftspolitische, pädagogische und kurioserweise auch auf die kommunikative Fremdsprachendidaktik. Gnutzmann (1997: 227) verweist beispielsweise darauf, dass Habermas' „mit Blick auf eine gesellschaftliche Utopie" entwickelte Theorie einen zwiespältigen Einfluss auf die kommunikative Fremdsprachendidaktik hatte. Diese Einschätzung teilt auch Schmenk (2005: 66), indem sie schreibt: „Diese "Sozialblindheit' des Kompetenzbegriffs Chomskys wird nun bei Habermas noch überhöht, indem sie zu einer gesellschaftlichen Sozialromantik gesteigert wird." Auch Kramsch setzt sich mit den Tendenzen der 70er und 80er Jahre kritisch auseinander, die $\mathrm{zu}$ einer Konzeption von Kommunikation geführt hat, die den erfolgreichen und möglichst reibungslosen Austausch von Information zwischen Individuen ins Zentrum der Aufmerksamkeit gerückt habe (vgl. Kramsch 1999: 42ff.). In ihrem Kern sei diese Sicht auf Sprache und Fremdsprachen, in Kramsch Worten:

[...] infused with a Western ideology, based on the enlightened belief in the democratizing value of symmetrical, unlimited turns-at-talk between like-minded speakers and hearers in free information exchanges, irrespective of their cultural backgrounds. (1999: 42)

Der Seitenhieb auf Jürgen Habermas' Überlegungen zu einem herrschaftsfreien Diskurs (vgl. Habermas 1971: 137) lässt sich hier nicht zu übersehen, auch wenn Kramsch ihn hier nicht expliziert.

\section{Kommunikativ-pragmatische Kompetenz als Leitziel des Fremdsprachenunterrichts? Kritik und Perspektiven}

Es ist auffallend, dass bei der Übertragung des Terminus „kommunikativpragmatische Kompetenz" auf den Bereich des institutionalisierten Fremdsprachenlernens insbesondere in der deutschen Fremdsprachendidaktik die Überlegungen von Habermas im Mittelpunkt standen, nicht die weitgefasste Definition von kommunikativer Kompetenz als Unterrichtsziel 
im Sinne von Hymes (1972), Canale und Swain (1980). Diese klare Präferenz war besonders deutlich in der ersten breit rezipierten Monographie „Kommunikative Kompetenz als oberstes Lernziel des Englischunterrichts“ von Hans E. Piepho (1974) zu erkennen, der die Idee der kommunikativen Kompetenz von Habermas für den Fremdsprachenunterricht als erster adaptiert hat. Seine eigene Definition von kommunikativer Kompetenz betont vor allem soziokulturelle Zielsetzungen, wie die Idee der Herrschaftsfreiheit und der Emanzipation der Lerner, die dem Lehrer gleichgesetzt sein sollten und ihm selbst lediglich eine Koordinationsfunktion im Unterricht zugewiesen wurde:

„Kommunikative Kompetenz bedeutet nämlich weder in der einen noch in der anderen Auslegung das Erreichen bestimmter Normen, sondern die Fähigkeit, sich ohne Ängste und Komplexe mit sprachlichen Mitteln, die man durchschaut und in ihren Wirkungen abschätzen gelernt hat, zu verständigen und kommunikative Absichten auch dann zu durchschauen, wenn sie in einem Code ausgesprochen werden, den man selbst nicht beherrscht und der nur partiell im eigenen Idiolekt vorhanden ist." (Piepho 1974: 9-10)

Die rein sprachlichen/grammatischen Komponenten von einer fremden Sprache und die Korrektheit sprachlichen Ausdrucks sollten im Kontext des Klassenzimmers weitgehend vernachlässigt werden. Piepho war auch der erste, der versucht hat, eine ideale, herrschaftsfreie Sprechsituation im Englischunterricht, d.h. in real existierenden Klassenzimmern, mit Hilfe von Rollenspielen einzuüben, was aus heutiger Perspektive sehr kontrovers wirkt bzw. als allzu naiver Glaube anzusehen ist.

Hier sei noch einmal hervorgehoben, dass weder Hymes' noch Habermas' Überlegungen zur kommunikativen Kompetenz direkt etwas mit dem didaktischen Prozess des Lehrens und Lernens fremder Sprachen zu tun haben. Sowohl Hymes ' Konzeption des Terminus scheint wenig hinreichend Begründung eines fremdsprachendidaktischen Lernziels zu sein als auch die Habermas`sche Projektion einer idealen Kommunikationssituation. Heutzutage wird die Tatsache, dass Hymes und Habermas als Kronzeugen für eine neue Ära bzw. für einen Paradigmawechsel in der Fremdsprachendidaktik gelten, sogar als Kuriosum angesehen. Es ist kaum möglich, so Kritiker, etwas als Lernziel zu erklären, das noch nicht einmal erhoben und systematisch beobachtet worden ist - nämlich kommunikative Kompetenz und ihre Realisierung in konkreten Kommunikationssituationen in unterschiedlichen sozialen und kulturellen Kontexten. Nicht der möglichst herrschaftsfreie Diskurs sollte im FU praktiziert werden, sondern jeder FU sollte Lernende sowohl mit 
linguistischer Kompetenz als auch mit kommunikativ-pragmatischer Kompetenz ausstatten. Auch wenn man sich weitgehend über die kommunikativ-pragmatische Kompetenz als Ziel des FU einig ist, d.h. über die Handlungsorientierung in authentischen Sprachlernsituationen, über den spielerisch endeckenden, kreativen Umgang mit der fremden Sprache, etc., soll diese keineswegs die reine Sprachkompetenz ausschließen (Götze 1991: 61-63, Götze/Helbig 2001: 12-30). Viele Studien zum Zweit- bzw. Fremdsprachenerwerb deuten darauf hin, dass die kommunikative Kompetenz sich bei längerem Sprachkontakt mit neative speakers mit Sicherheit verbessert, was aber nicht unbedingt auch für die grammatische Korrektheit gelten muss. Man kann z.B. während eines längeren Auslandsaufenthaltes lernen, die Fremdsprache in einer Vielzahl unterschiedlicher Lebenssituationen effektiv, flüssig $\mathrm{zu}$ verwenden; die Beherrschung des Sprachsystems wird dadurch aber nicht zwangsläufig verbessert.

Im Rahmen der polnischen Glottodidaktik wurde von Anfang an eine Position repräsentiert, die auf die Notwendigkeit einer unterrichtlichen Progression von rein sprachlicher Kompetenz hin zur Kommunikationsund Kulturkompetenz verweist. Nach Grucza (1988: 309-331, 1993: 29ff.) macht die Kenntnis der jeweiligen Sprache im engeren Sinne, d.h. die einwandfreie Aneignung der reinen Sprachkompetenz, den Kern einer jeden menschlichen Kommunikations- und Kulturkompetenz aus und sollte demzufolge als der zentrale Bezugspunkt der Lehrfunktion eines jeden Sprachlehrers angesehen werden: „Wer sich sprachlicher Äußerungen kommunikativ bedienen will, muss sich zunächst die Fähigkeit aneignen (a) sie $\mathrm{zu}$ erzeugen, d.h. ihre Strukturen zu bilden und mit konkreten Wörtern $\mathrm{zu}$ füllen, (b) die erzeugten Äußerungen $\mathrm{zu}$ identifizieren und $\mathrm{zu}$ interpretieren" (Grucza. 2002: 231-244). Die Sprache im generativen Sinne muss also jeder Lerner aufgrund der ihm zugelieferten Äußerungen selbst rekonstruieren. Er ist sowohl auf die Form der Äußerung als auch auf die sog. Substanz angewiesen. Aufgabe der unterrichtlichen Fremdsprachenvermittlung ist es, in erster Linie die Voraussetzungen für den Aufbau der formalen Kompetenz zu schaffen. Unter dem Einfluss von Äußerungen, die von der jeweiligen fremdsprachlichen Umwelt erfolgen, wird die jeweilige Kernkompetenz erst konkretisiert. F. Grucza (2017: 124) argumentiert auf folgende Art und Weise: „Es stimmt jedoch nicht, dass sich die Sprachkompetenz ausschliesslich unter dem Einfluss fremder Äusserungen entfaltet." Laut Grucza entfaltet jeder Sprachlerner, auch Fremdsprachenlerner eigene formale Kompetenz aus eigener Kraft und dann wird diese 
entfaltete/angeeignete Sprachkompetenz aus eigener Initiative weiter entwickelt/ausgebaut.

Mit die Einführung der kommunikativen Kompetenz in die theoretische Diskussion und in die schulische Praxis werden nur selten zufriedenstellende Ergebnisse erreicht, worauf mit Recht Grucza verweist (2002: 231): „Dies führte zu einer Überbetonung des kommunikativen Umgangs mit Äußerungen und zur Überbewertung der Rolle der sprachlichen Diskurs- und Handlungskompetenz bei gleichzeitiger Unterbewertung der generativproduktiven Kompetenz.“ M. Müller-Verweyen (2002: 429) spricht sogar von einer „Deformation des kommunikativen Fremdsprachenparadigmas“ und verweist auf eine Überbewertung der face to face Situation im FU sowie auf die Tatsache, dass der kommunikative Ansatz das Vorwissen bzw. die Vorerfahrung der Lernenden und die Rolle reflexiver Prozeduren unterschätzte und dabei mit einem eingeschränkten Begriff von Authentizität arbeitete. Der kommunikativen Didaktik wird auch vorgeworfen, dass sie bislang versäumt hat, Sprache konsequent als Diskurs zu betrachten. Die kommunikative Fremdsprachendidaktik wurde somit im Laufe der Zeit nach und nach immer stärker auf den Bereich der Mündlichkeit reduziert und sich in zunehmendem Maße auf Aspekte der Funktionalität, Effizienz und Messbarkeit ausgerichtet. Kramsch (2006: 250) schreibt dazu:

In the educational world, communication has been slowly resignified to mean the ability to exchange information speedily and effectively and to solve problems, complete assigned tasks, and produce measurable results.

Die Fixierung auf die Lösung von kommunikativen Aufgaben hat Kramsch zufolge dazu geführt, dass heutzutage viel zu selten Sinn und Zweck der Aufgaben selbst hinterfragt werde: „What often needs to be negotiated nowadays is not how to achieve the task, but the nature and the purpose of the task itself" (Kramsch (2006: 250). Mit Recht hat sie bemerkt, dass besonders problematisch war, unterrichtliche Kommunikation als Streben nach Konsens zu entwerfen - und, „wie man ergänzen darf, damit dem Habermas'schen Ideal zu folgen (vgl. Kramsch 1999: 43).

\section{Ein komplementäres Konzept als Herausforderung für die Glottodidaktik im 21. Jahrhundert}

Ein besonderer Fokus gilt in dem vorliegenden Artikel den interdisziplinären Bezügen der kommunikativen Didaktik, die sich dadurch auszeichneten, dass 
hier eine große Offenheit existierte, was Erkenntnisse bzw. Einsichten und Ansätze aus anderen sog. Bezugsdisziplinen betraf. Die Fremdsprachendidaktiker griffen auf Theorien, Modelle und Begriffe zahlreicher anderer Fachbereiche zurück, manche wurden einfach unbegründet ignoriert. Mit anderen Worten, die kommunikative Fremdsprachendidaktik versuchte von vielen Bezugsdisziplinen, die sie in entscheidender Weise inspiriert und weitgehend mitgeprägt haben, zu profitierten. $\mathrm{Da}$ sie jedoch nicht eindeutig fremdsprachendidaktisch ausgerichtete waren, ist nicht selten sogar zu einer Trivialisierung kommunikativer Kompetenz im FU gekommen. Dies lässt sich u.a. auf das eingeschränkte Sprach-, Kommunikations- und Kulturverständnis innerhalb der Fremdsprachendidaktik zurückführen. In erster Linie steckte eine solche Reduktion im Verständnis der Natur der menschlichen Sprache, was maßgeblich $\mathrm{zu}$ vielen Misserfolgen in der fremdsprachendidaktischen Praxis geführt hat.

Die polnische Glottodidaktik ist anthropozentrisch orientiert und legt in ihrem Forschungsprogramm ein besonderes Augenmerk auf den Menschen in der Funktion des Sprachlerners und Sprachlehrers². Die Erforschung der konstitutiven Eigenschaften (facultas linguarum, d.h. Sprach(erwerbs)fähigkeiten, Sprachverarbeitungsfähigkeiten, Sprachwissen, etc.), die den kommunikativpragmatischen Kompetenzen der Sprachlerner und der Sprachlehrer zugrunde liegen und demzufolge als wichtige Faktoren der generativen Ausstattung dieser Objekte bzw. Subjekte zu verstehen sind, wird als eine zentrale Aufgabe der Glottodidaktik definiert. Mit anderen Worten, die aktuelle glottodidaktische Forschung konzentriert sich vorwiegend auf die Ausarbeitung einer Sprachlehrer- und Sprachlernertheorie, die für die wissenschaftliche Fundierung des institutionellen Lehrens und Lernens fremder Sprachen in Polen relevant sein wird. Eine solche wissenschaftliche Theorie soll eine explikative und eine prognostische Funktion erfüllen. Demzufolge wird zwischen dem reinen (beschreibend-erklärenden) und dem angewandten (normativ-direktiven) Aspekt einer Theorie unterschieden. F. Grucza $(2010,2017)$ vertritt den Standpunkt, dass sich die Glottodidaktik keinesfalls nur auf die theoretische Begründung ihrer Erkenntnisprodukte beschränken kann, sondern auch ihre praktische Bestätigung bzw. Widerlegung anstreben muss. Angewandte Erkenntnisse der Glottodidaktik sollen aber von jeglicher praktischen Sprachdidaktik streng getrennt werden.

2 F. Grucza, Glottodydaktyka: nauka - praca naukowa - wiedza. In: Glottodydaktyka 20, 2006, 19-40. 
Zusammenfassend lässt sich festhalten, dass die pragmatischkommunikative Kompetenz für das Lehren und Lernen fremder Sprache nicht mehr eine Schlüsselrolle spielt bzw. spielen sollte; vielmehr sollte im Lichte der neuen Herausforderungen für die Fremdsprachendidaktik im 21. Jahrhundert ein komplementäres Konzept aufgebaut werden, in dem der einwandfreie Erwerb der reinen formal-grammatischen Kompetenz den Kern einer jeden Kommunikations- und Kulturkompetenz mit ihrem universalistischen Verständnis von Kultur ausmachen sollte und demzufolge als der zentrale Bezugspunkt für die Bestimmung relevanter Bezugsdisziplinen für die Fremdsprachendidaktik angesehen werden sollte.

\section{LITERATURVERZEICHNIS}

Apel, K.-O. (Hg.) (1975). Sprachpragmatik und Philosophie. Frankfurt/M: Suhrkamp.

Appel, J. (2017). Der kommunikative Denkstil, Zeitschrift für Fremdsprachenforschung 281 , 77-100.

Austin, J. (1962. How to do things with words. Oxford: Clarendon Press.

Austin, J. (1972). Zur Theorie der Sprechakte. Dt.v. savigny. Stuttgart: Reclam.

Austin, J. (1975). Sinn und Sinneserfahrung. Stuttgart: Suhrkamp.

Baker, G. \& Hacker P. (1984): Language, sense and nonsense: a critical investigation into modern theories of language. Oxford: Blackwell.

Canale, M. \& Swain, M. (1980). Theoretical Bases of Communicative Approaches to Second Language Teaching and Testing. Applied Linguistics 1, 1-47.

Chomsky, N. (1975). Reflections of language. New York: Pantheon.

Chomsky, N. (1981). Lectures on Government and Binding. Dordrecht: Foris.

Chomsky, N. (1986). Knowledge of Language. Its Nature, Origin, and Use. New York: Praeger.

Chomsky, N. (1988). Language and Problems of Knowledge. Cambridge, Mass.: MIT.

Chomsky, N. (1995). The Minimalist Program. MA/London, Cambridge Mass.

Chomsky, N. (2002). On Nature and Language. Cambridge.

Chomsky, N. (2011). Language and other cognitive systems: What is special about language? In: Language Learning and Development, 7(4), 263-278.

Chomsky, N. (2016). Was für Lebewesen sind wir? Berlin: Suhkamp Verlag.

Ellis, R. \& Shintani, N. (2014). Exploring Language Pedagogy through Second Language Acquisition Research. London/New York: Routledge.

Fanselow, G. \& Felix, S. (1987). Sprachtheorie. Eine Einführung in die Generative Grammatik. Tübingen: Francke.

Götze, L. (1991). Grammatik und Kommunikation - ein Widerspruch? DaF 28/3 161-163.

Götze, L. \& Helbig, G. (2001). Linguistischer Ansatz. In: Helbig, G./ Götze, L. / Henrici, G. / Krumm, H.-J. (Hg.) Deutsch als Fremdsprache. Ein internationales Handbuch. 1. Halbband. Berlin/New York: de Gruyter, 12-30.

Gnutzmann, C. (1997). Language Awareness. Geschichte, Grundlagen, Anwendung. Praxis des neusprachlichen Unterrichts, 3, 227-236. 
Gnutzmann, C. /Königs, F. \& Küster, L. (2011). Fremdsprachenunterricht und seine Erforschung. Ein subjektiver Blick auf 40 Jahre Forschungsgeschichte und auf aktuelle Forschungstendenzen in Deutschland. Fremdsprachen Lehren und Lernen, 40 (1), 5-28.

Grewendorf, G. (1995). Sprache als Organ. Sprache als Lebensform. Anhang Interview mit Noam Chomsky: Über Linguistik und Politik. Frankfurt/M.: Suhrkamp.

Grewendorf G., Hamm F. \& Sternefeld W. (1993). Sprachliches Wissen. Frankfurt/M.: Suhrkamp.

Grucza, F. (1988). Zum Begriff der Sprachkompetenz, Kommunikationskompetenz und Kulturkompetenz. In: Honsza, N./ Roloff, H.-G. (Eds.): Daß eine Nation die andere verstehen möge. Festschrift für M. Szyrocki zu seinem 60. Geburtstag (= Chloe. Beihefte zu Daphnis Bd. 7) Amsterdam, 309-331.

Grucza, F. (2002). Zu den Forschungsgegenständen der Linguistik und der Glottodidaktik. Zum Wesen menschlicher Sprachen und zu ihren Funktionen. In: Barkowski, H./ Faistauer, R. (Eds.) in Sachen Deutsch als Fremdsprache. Baltmannsweiler: Schneider Verlag, 231-244.

Grucza, F. (2006). Glottodydaktyka: nauka - praca naukowa - wiedza, Glottodydaktyka, 20, 19-40.

Grucza, F. (2012). Lingwistyka i kulturologia antropocentryczna. Warszawa.

Grucza, F. (2017). Dzieła Zebrane. Tom 6. O nauczaniu języków i glottodydaktyce. Warszawa.

Grucza, F. \& Krumm, H.-J. / Grucza, B. (1993). Beiträge zur wissenschaftlichen Fundierung der Ausbildung von Fremdsprachenlehrern, Warszawa: Wydawnictwo Uniwersytetu Warszawskiego.

Fanselow, G. \& Felix, S. (1987). Sprachtheorie. Eine Einführung in die generative Grammatik. Bd.1: Grundlagen und Zielsetzungen. Tübingen: Francke.

Hartshorne, C. \& Weiss, P. (Hg.) (1933-34). Collected Papers of Charles Sanders Peirce. Bd. 5, 1905, Cambridge Mass.

Hookway, C. (2012). The Pragmatic Maxim. Essays on Peirce and Pragmatism. Oxford: Oxford University Press.

Habermas, J. (1971). Vorbereitende Bemerkungen zu einer Theorie der kommunikativen Kompetenz. In: Habermas, J. / Luhmann, N. (Hg.), Theorie der Gesellschaft oder Sozialtechnologie - Was leistet die Systemforschung? Frankfurt/M: Suhrkamp, 101-141.

Habermas, J. (1976). Was heißt Universalpragmatik? In: Apel, K.-O. (Hg.), Sprachpragmatik und Philosophie. Frankfurt/M.: Suhrkamp, 174-272.

Habermas, J. (1995). Theorie des kommunikativen Handelns. Frankfurt/M.: Suhrkamp.

Hymes, D. (1972). On Communicative Competence. In: Pride, J. B./ Holme, J. (Hg.), Sociolinguistics. Selected Readings. Harmondsworth: Penguin, 269-293.

Humboldt, W. von (1979). Werke in fünf Bänden. Bd. III, Schriften zur Sprachphilosophie. Darmstadt: Wissenschaftliche Buchgesellschaft (Estauflage 1836).

Kapuściński, R. (1994). Macht und Ohnmacht. NZZ_FOLIO, Oktober 1994, 32-

Kienzel, W. (2007). Ludwig Wittgensteins «Philosophische Untersuchungen». Darmstadt: WBG.

Kramsch, C. (1999). Thirdness: The Interkultural Stance. In: Vestergard Torben, Language, Culture and Identity. Aaborg, Denmark: Aalborg University Press, 41-58.

Kramsch, C. (2000). Second Language Acquisition, Applied Linguistics, and the teaching of foreign languages. The Modern Language Journal 84/3, 311-326.

Kramsch, C. (2000a). A New Field of Research: SLA-Applied Linguistics. Modern Language Association 115/7, 1978-80.

Kramsch, C. (2006). From Communicative Competence to Symbolic Competence. The Modern Language Journal 90, 244-266. 
Kramsch, C. (2011). Theorizing translingual/transcultural competence. In: Levine, G./ Phipps, A. (Hg.), Critical and Intercultural Theory and Language Pedagogy. Boston: Heinle.

Mead, G. H. (1934/dt. 1968). Mind, Self and Society from the Standpoint of a Social Behaviorist. Chicago: Chigaco Univ. Press./Dt. Geist, Identität und Gesellschaft - aus der Sicht des Sozialbevaviorismus. Frankfurt/M: Suhrkamp.

Legutke, M. / Schart, M. (2016). Fremdsprachliche Lehrerbildungsforschung. Bilanz und Perspektiven. In: Ders. (Hg.), Fremdsprachendidaktische Professionsforschung: Brennpunkt Lehrerbildung. Tübingen: Gunter Narr, 9-46.

Morris, W. Charles (1938/dt. 1988). Foundations of the Theory of Signs. Dt. Grundlagen der Zeichentheorie. Frankfurt/M.: Suhrkamp.

Morris W. Charles (dt. 1973). Zeichen Sprache und Verhalten. Düsseldorf: Schwann.

Müller-Verweyen, M. (2002). Europe matters. Eine Strategie zur Förderung von Deutsch als Fremdsprache. Ein Japan im Rahmen des Kontextes ,Europa'. Jahrbuch Deutsch als Fremdsprache 28, 429-442.

Nagl, L. (1998). Pragmatismus. Frankfurt/M: Suhrkamp/ New York: Campus Verlag.

Oehler, K. (1993). Charles Sanders Peirce. München: Beck.

Peirce, C. S. (1905). Collected Papers of Charles Sanders Peirce. Bd. 5 hg. von Charles Hartshorne u. Paul Weiss, Cambridge Mass 1933-34, Cambridge MA: Harvard University.

Peirce, C. S. (1968). Über die Klarheit unserer Gedanken. Hg. v. K. Oehler, Frankfurt/M.: Kostermann.

Peirce, C. S. (1991). Schriften zum Pragmatismus und Pragmatizismus, (Hg), Apel, K.-O./ (übers. von G. Wartenberg), 2.Aufl., Frankfurt /M.: Ulstein.

Peirce Ch. S. (1993). Phänomen und Logik der Zeichen. (hrsg. und übers. von H. Pape) Frankfurt/M.

Piepho H.-E. (1974). Kommunikative Kompetenz als übergeordnetes Lernziel des Englischunterrichst. Dornburg: Frickhofen.

Pinker, S. (1997). How the Mind Works. (2. Aufl. 2009). New York: W. W. Norton \& Company.

Pinker, S. (1998). Sprachinstinkt. Wie der Geist die Sprache bildet. München: Knaur.

Portmann P. (1981). Kommunikation als Problem der Sprachdidaktik. Untersuchungen zur Integration kommunikationstheoretischer Modelle in einigen neueren Theorien des Sprachunterrichts. Tübingen: Narr.

Sadownik, B. (1997). Glottodidiaktische und psycholinguistische Aspekte des Fremdsprachenerwerbs: Lernerperspektive. Lublin: Wydawnictwo Uniwersytetu Marii Curie-Skłodowskiej.

Sadownik, B. (2010). Modulare Architektur der menschlichen Sprachfähigkeit. Sprachliche und neurobiologische Dimensionen. Wydawnictwo Uniwersytetu Marii CurieSkłodowskiej.

Sadownik, B. (2019). Menschliche Sprachfähigkeit und ihre neurobiologischen Korrelate. Reflexionen aus glottodidaktischer Sicht. Linguistics Papers 26/1, 155-168.

Schmenk, B. (2005). Mode, Mythos, Möglichkeiten oder ein Versuch, die Patina des Lernziels, kommunikative Kompetenz' abzukratzen. ZFF 16 (1), 57-88.

Schmenk, B. (2007). Kommunikation ist alles. Oder? Wider die Trivialisierung des Kommunikativen im kommunikativen Fremdsprachenunterricht. DaF 44 (3), 131-139.

Schmenk, B. (2015). Eine sonderbare Spezies: Fremdsprachendidaktik. Eine (?) Disziplin (?) an den Schnittstellen von akademischen, bildungspolitischen, gesellschaftlichen und erzieherischen Forschungs- und Wirkungsfeldern. In: Doff, S. / Grünewald, A. (Hrsg.). 
WECHSEL-Jahre? Wandel und Wirken in der Fremdsprachenforschung. Trier: Wissenschaftlicher Verlag, 5-16.

Schmenk, B. (2019). Zum Spannungsfeld der Fremdsprachendidaktik und ihrer Bezugswissenschaften. In: Wilden, E./Rossa, H. (Hg.), Fremdsprachenforschung als interdisziplinäres Projekt. Berlin, Bern, Bruxelles, New York, Oxford, Warszawa, Wien. Frankfurt/M.: Peter Lang.

Schneider, G. / North, Brian (2007). Fremdsprachen können - was heisst das? Skalen zur Beschreibung, Beurteilung und Selbsteinschätzung der fremdsprachlichen Kommunikationsfähigkeit. Chur/Zürich: Verlag Rüegger.

Searle, John R. (1969/dt.1971). Speech Acts. An Essay in the Philosophy of Language. Cambridge: Cambridge University Press. / Dt. Sprechakte. Ein sprachphilosophischer Essay. Franfurt/M.: Suhrkamp.

Searle, John R. (1971/1976). A Taxomy of Illocutionary Acts. Reprint Trier: L.A.U.T.

Searle, John R. (1976). The rule of the language game. The Times Literary supplement 1118 (10.9.1976).

Searle, John R. (1979/dt.1982). Ausdruck und Bedeutung. Frankfurt/M.: Suhrkamp.

Searle, John R. (1980). Rules and causation. Commentary on Chomsky`s Rules and Representations. Behavioral and Brain Sciences 3, 37-38.

Searle John R. (1984/dt. 1986). Geist, Hirn und Wissenschaft. Frankfurt/M.: Suhrkamp.

Tomasello, M. (2004). What kind of evidence could refute the UG hypothesis? Studies in Language 28, 642-644.

Tomasello, M. (2006). Die kulturelle Entwicklung des menschlichen Denkens. Zur Evolution der Kognition. Frankfurt a. M.

Tomasello, M. (2009). Universal Grammar is dead. Behavioral and Brain Sciences 32(5), 470-471.

Tomasello, M. (2011). Ursprünge der menschlichen Kommunikation. Frankfurt.

Tomasello, M. (2014). Eine Naturgeschichte des menschlichen Denkens. Berlin.

Trettenbrein, P. (2015). The "grammar" in Universal Grammar: A biolinguistic clarification. Questions and Answers in Linguistics 2 (1), 1-10.

Wittgenstein, L. (1953/dt. 1984). Philosophical Investigation. Oxford: Basil Blackwell/ Dt. Philosophische Untersuchungen, Werke, Bd.1. Frankfurt/M.: Suhrkamp. 
